\title{
Attenuation and delay of remote potentials evoked by direct electrical stimulation during brain surgery
}

Anthony Boyer ${ }^{\mathrm{a}}$, Hugues Duffau ${ }^{\mathrm{b}, \mathrm{c}}$, Emmanuel Mandonnet ${ }^{\mathrm{d}}$, Marion A. Vincent ${ }^{\mathrm{a}, \mathrm{e}}$, Sofiane Ramdani $^{\mathrm{f}}$, David Guiraud ${ }^{\mathrm{a}}$ and Francois Bonnetblanc ${ }^{\mathrm{a}, \mathrm{g}, \mathrm{h}}$

${ }^{a}$ CAMIN, INRIA, LIRMM, Université de Montpellier, 34090 Montpellier, France.

b "Plasticité cérébrale, cellules souches neurales et tumeurs gliales ", INSERM U1051, Institut des Neurosciences de Montpellier, 34295 Montpellier, France.

${ }^{\mathrm{c}}$ Département de Neurochirurgie, Centre Hospitalier Régional Universitaire de Montpellier Gui de Chauliac, 34295 Montpellier, France.

${ }^{\mathrm{d}}$ Département de Neurochirurgie, Hopital Lariboisière, Paris, France.

${ }^{\text {e }}$ SCALab - Sciences Cognitives et Sciences Affectives, CNRS, UMR 9193, Université de Lille, F-59000 Lille, France.

${ }^{\mathrm{f}}$ IDH, LIRMM, Université de Montpellier, 34095 Montpellier, France.

${ }^{\mathrm{g}}$ Institut Universitaire de France, 75231 Paris, France.

h "Cognition, Action et Plasticité Sensorimotrice", INSERM U1093, UFR STAPS, Université Bourgogne Franche-Comté, 21078 Dijon, France.

Corresponding authors: david.guiraud@inria.fr and francois.bonnetblanc@inria.fr 


\begin{abstract}
Background: Direct electrical stimulation (DES) is used to perform functional brain mapping during awake surgery but its electrophysiological effects remain by far unknown.

Hypothesis: DES may be coupled with the measurement of Evoked Potentials (EPs) to study the conductive and integrative properties of activated neural ensembles and probe the spatiotemporal dynamics of short- and long- range networks.

Methods: We recorded ECoG signals on two patients undergoing awake brain surgery and measured EPs on functional sites after cortical stimulations, using combinations of stimulation parameters.

Results: EPs were similar in shape but delayed in time and attenuated in amplitude when elicited from a different gyrus or remotely from the recording site. We were able to trigger remote EPs using low stimulation intensities.
\end{abstract}

Conclusion: We propose different activation and electrophysiological propagation mechanisms following DES based on activated neural elements.

\title{
Keywords (5/6)
}

low-grade glioma - awake brain surgery - direct electrical stimulation - evoked potentials - electrocorticography

\section{Introduction}

Direct electrical stimulation (DES) is used to perform intraoperative functional mapping of the brain and guide tumor resection during awake neurosurgery of low-grade gliomas. It generates transient behavioral/cognitive disturbances allowing the identification of both cortical areas and subcortical white matter pathways, which are essential to the function (Duffau 2015, Mandonnet et al. 2010). DES electrophysiological effects remain poorly understood and the recording of Evoked Potentials (EPs) may be used to study the conductive and integrative properties of the neural ensembles being (in)directly affected (Vincent et al. 2016). Three types of cortical EPs should be differentiated: (1) the Direct Cortical Response (DCR), recorded in the immediate vicinity (same gyrus) of the cortical stimulation site and commonly occurring with a $20 \mathrm{~ms}$ delay; (2) the Cortico-Cortical Evoked Potential (CCEP), elicited by the physiological propagation through white matter tracts from the stimulated cortical area towards the remote recording site, around $30 \mathrm{~ms}$ after the stimulation and (3) the Axono-Cortical Evoked Potential (ACEP), recorded cortically after the stimulation of underlying white matter tracts (Vincent et al. 2017).

The DCR typically consists of a negative deflection called the primary negative potential (N1) which peaks between 15 and $25 \mathrm{~ms}$ after the stimulation onset and is believed to result from the spatiotemporal summation of post-synaptic potentials at the apical dendrites of pyramidal cells. A stronger stimulus intensity may elicit more complex EPs with spikes of positive polarity and brief duration (approximately $5 \mathrm{~ms}$ ) appearing in the initial phase of the 
response, and followed by the N1 component. This early positive deflection, the P0 component, has been ascribed to serial "all or none" discharges in the soma of cortical pyramidal neurons and could be illustrative of the summation of synchronous action potentials (Goldring et al. 1961, Li and Chou 1962, Goldring et al. 1994, Vincent et al. 2017). On the other hand, the electrogenesis of CCEP and ACEP remain ambiguous and the "spread of activity in the cerebral cortex" as initially investigated by Adrian (1936) is mostly unknown (Matsumoto et al. 2004, Yamao et al. 2014).

DES coupled with the recording of EPs could be used to assess the electrophysiological status of cortical sites of interest and more particularly to probe the spatiotemporal connectivity in short- and long- range networks. We hypothesized that the amplitude and latency of EPs may be dependent on the propagation mechanisms (i.e. intracortical vs. subcortical). More specifically, the N1 should be attenuated and, more importantly, delayed, when measured in a different gyrus or lobe because of the additional conduction time and divergence of association fibers.

\section{Material and methods}

Two patients underwent an awake brain surgery of a low-grade glioma. The first patient (P1) was a 41-years old man with a glioma located within the left fronto-temporoinsular region. The second patient (P2) was a 50-years old woman with a glioma located within the left paralimbic region. The study (UF 965, $\mathrm{n}^{\circ}$ 2014-A00056-43) was approved by the local ethics committee and patients signed an informed consent. ECoG data were recorded intra-operatively, after the tumor resection and under general anesthesia. Two 4-electrode ECoG strips ( $2.5 \mathrm{~mm}$ platinium contacts, $10 \mathrm{~mm}$ spaced, DIXI, France) were positioned on the brain surface of both patients (see B and D). ECoG signals were recorded using differential configurations for both strips, sampled at $10 \mathrm{kHz}$ (PowerLab, ADInstrument) and band-pass filtered from $0.5 \mathrm{~Hz}$ to $1 \mathrm{kHz}$. The front-end amplifier (g.BSamp, G.tec, Austria) was grounded to a patch-electrode located on the right acromion and signals were recorded with a gain of 1,000. Two functional sites identified during the awake mapping were stimulated using biphasic constant-current square wave delivered thanks to a bipolar probe (0.5 mm diameter electrode tips, $5 \mathrm{~mm}$ apart) (Nimbus, Innopsys, France). At each stimulation site, 3 stimulation conditions were tested and repeated twice consecutively. The choice of the stimulation parameters was based on previous work which proposed that shortened Pulse Width (PW) and increased Inter-Electrode Distance (IED) may help recruiting different neural elements (Boyer et al. 2018): (1) $\mathrm{PW}=1 \mathrm{~ms}$, IED $=0.5 \mathrm{~cm}$; (2) PW $=0.5 \mathrm{~ms}, \mathrm{IED}=0.5 \mathrm{~cm}$; (3) $\mathrm{PW}=0.5 \mathrm{~ms}, \mathrm{IED}=1 \mathrm{~cm}$. The stimulation intensity was set to 2 $\mathrm{mA}$ and frequency was chosen so it allowed long enough time-window to record EPs, especially the $\mathrm{N} 1$ component, while keeping the stimulation duration as short as possible (10 and $9 \mathrm{~Hz}$ for P1 and P2 respectively). Mean EPs traces were obtained by averaging ECoG signals time-locked to the DES onset. The number of stimuli available depended on the stimulation frequency and its duration, which ranged from 4 to $8 \mathrm{~s}$. The baseline of individual stimulus was set before averaging, using the mean value of the last $10 \mathrm{~ms}$ preceding each DES artifact. Also, mean EPs obtained using the first and last 15 repetitions were compared in order to verify for EP stability over time, controlling for possible bias induced by the $9-10 \mathrm{~Hz}$ stimulation, especially progressive polarization. Finally, 99\% confidence intervals (Standard Error of the Mean) were estimated for each DCR as they showed measurable EPs after each stimulus. 


\section{Results}

The experiment lasted 12 and 9 minutes for $\mathrm{P} 1$ and $\mathrm{P} 2$ respectively. In total, 12 stimulations were performed on each patients ( 3 stimulation conditions, repeated twice, on 2 sites) and cortical responses were recorded between each electrodes of both strips ( 6 channels measuring differential signals) resulting in 72 traces per patient. 35\% of traces (31 for P1 and 19 for P2) were not exploitable because of faulty measurement and/or very poor signal-tonoise ratio. $30 \%$ of traces (22 for $\mathrm{P} 1$ and 22 for $\mathrm{P} 2$ ) did not show noticeable EPs after averaging. Finally, 35\% of traces (19 for P1 and 31 for P2), showed EPs after averaging, and DCRs were sometimes distinguishable on raw signal.

Overall, all measured EPs were similar in shape but delayed in time and largely attenuated in amplitude when elicited from a different gyrus or lobe than the one being recorded. More importantly, we were able to measure EPs triggered by both close and remote stimulations for a few recording locations. The recording channels discussed and presented in Fig.1, are the ones for which we were able to record both DCRs and CCEPs, elicited by intraand extra- gyral DES respectively.

This was the case for P1 when stimulating Wernicke's area (S1) which produced local DCRs and elicited CCEPs over the ventral premotor cortex and inversely (see panels A and B). For P2, we recorded DCRs and CCEPs over the precentral gyrus after stimulating respectively the ventral premotor cortex (S2) and the middle part of the superior temporal gyrus (S1), despite the presence of the Sylvian fissure and the operative cavity in-between (see D). Interestingly for this patient, we also measured CCEPs over the superior temporal gyrus when stimulating the precentral gyrus (S2) but abnormally delayed EPs when stimulating residual temporal tissue next to the operative cavity (S1) (see C). Regarding stimulation parameters, we were able to trigger long distance CCEPs when using $1 \mathrm{~cm}$ IED with regard to other stimulation conditions which may not induce EPs (see B and D) but they also appeared altered in both latency and amplitude when compared to other existing CCEPs (see A and C). 

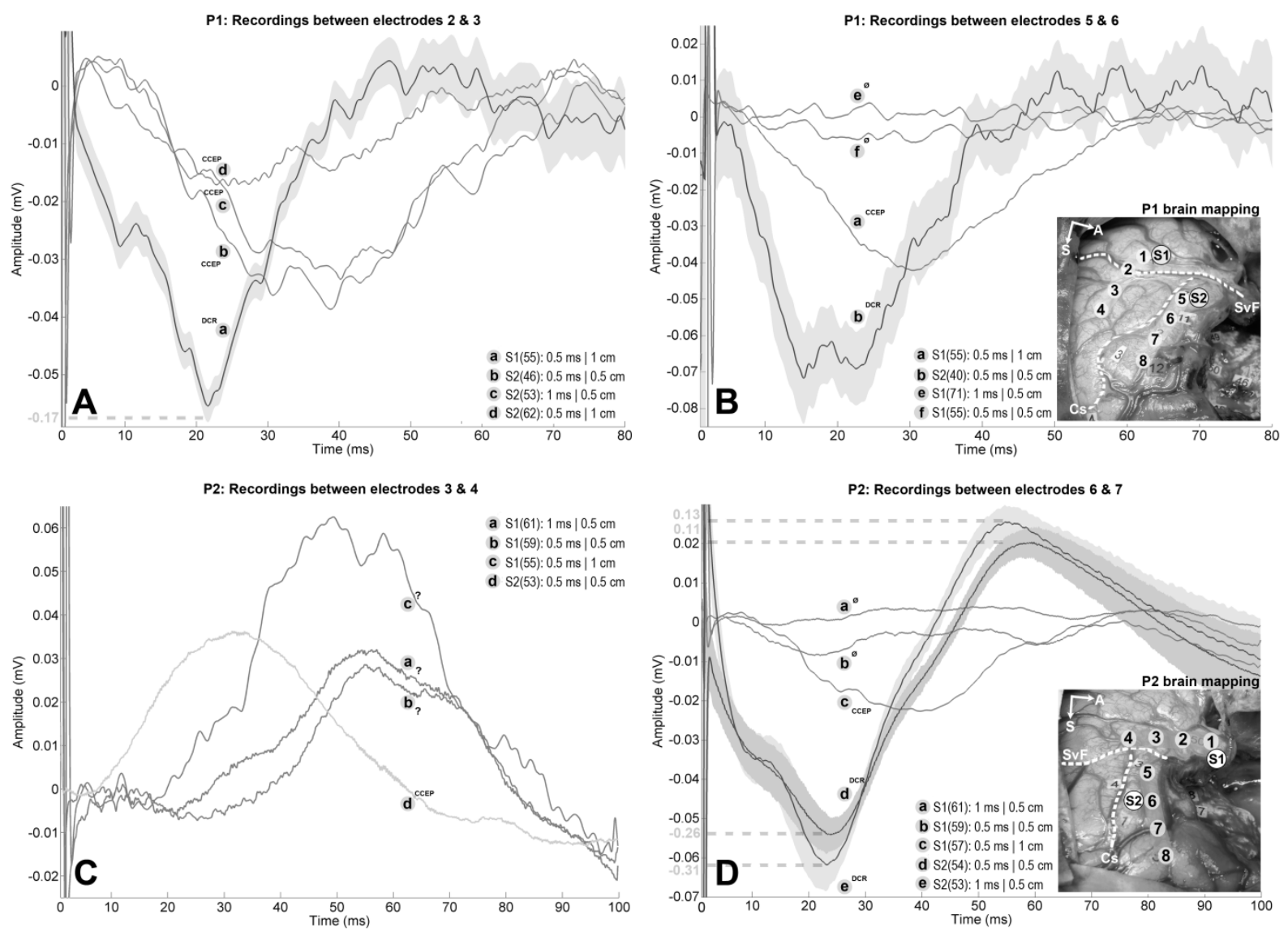

P1 and P2 brain mappings: Pictures illustrating the stimulation sites (S1, S2) and ECoG positioning with respect to the initial $60 \mathrm{~Hz}$ cortical brain mapping (numbered paper tags). Electrodes of both ECoG strips are numbered from 1 to 4 and from 5 to 8 . The Sylvian fissure and central sulcus are highlighted by a white dashed lines and annoted "SyF" and "Cs" respectively. For P1, experimental DES was applied on: (1) the Wernicke's area (S1), associated with complete anomia; (2) the ventral premotor cortex (S2), which led to movement and counting interruptions. Strip 1 spans over both temporal and parietal lobe with: electrode 1 over the most posterior part of the superior temporal gyrus; electrode 2 over the Sylvian fissure; electrodes 3 and 4 over the adjacent supramarginal gyrus. Strip 2 spans over the precentral gyrus with: electrodes 5 to 7 over the ventral premotor cortex; electrode 8 is bordering with the most posterior part of the partially resected dorsolateral prefrontal cortex. For P2, experimental DES was applied on: (1) the middle part of the superior temporal gyrus (S1) which led to complete anomia; (2) the precentral gyrus (S2), which induced articulatory disorders. Strip 1 spans over the superior temporal gyrus with: electrodes 1 and 2 over its middle third; electrodes 3 and 4 over its most posterior part. Strip 2 spans over the precentral and dorsolateral prefrontal gyri with: electrodes 5 and 6 over the ventral premotor cortex; electrodes 7 and 8 are respectively bordering and within the adjacent dorsolateral prefrontal cortex. Tumor was about $164 \mathrm{~cm}^{3}$ for P1 and $150 \mathrm{~cm}^{3}$ for P2. The number of averaged stimuli is reported within parentheses for each trace. 99\% confidence interval estimated for DCRs are represented by grey surfaces to demonstrate that CCEPs do not belong to them. Additional traces corresponding to variations of stimulation parameters were added if available, regardless of the presence of EPs. A: Differential recordings between electrodes 2 and 3 for P1 while stimulating S1 (-170 $\mu \mathrm{V}, 21 \mathrm{~ms}$ delay) and S2 (amplitudes ranging from $-40 \mu \mathrm{V}$ to $-17 \mu \mathrm{V}$, delays ranging from $25 \mathrm{~ms}$ to $38 \mathrm{~ms}$ ). EPs following $\mathrm{S} 2$ stimulation are CCEPs because of the presence of the central fissure between the stimulation and recording sites. The EP measured after stimulating S1 is ambiguous because electrode 2 lies on the Sylvian fissure, but the short latency and enhanced amplitude with regard to the CCEPs suggest a DCR. Note the dashed line indicating a different amplitude scale for the DCR, which was reduced by a factor 3 for visualization purposes. B: Differential recordings between electrodes 5 and 6 for P1 while stimulating $\mathrm{S} 2(-75 \mu \mathrm{V}, 20 \mathrm{~ms}$ delay) and $\mathrm{S} 1(-44 \mu \mathrm{V}, 30 \mathrm{~ms}$ delay). EP following S1 stimulation is a CCEP because of the presence of the Sylvian fissure between the stimulation and recording sites. EP following S2 stimulation should be viewed as DCR as it was recorded on the same gyrus and it showed shorter latency and enhanced amplitude in comparison with the CCEP. C: Differential recordings between electrodes 3 and 4 for P2 while stimulating S1 (amplitudes ranging from $+29 \mu \mathrm{V}$ to $+62 \mu \mathrm{V}$, delays 
ranging from $52 \mathrm{~ms}$ to $62 \mathrm{~ms})$ and $\mathrm{S} 2(+36 \mu \mathrm{V}, 32 \mathrm{~ms}$ delay). EP following S2 stimulation is a CCEP because of the presence of the Sylvian fissure between the stimulation and recording sites. EPs following S1 stimulations should be viewed as DCRs as they are recorded on the same gyrus but the latencies and amplitudes appeared unusual. EPs are positive because of differential measure. D: Differential recordings between electrodes 6 and 7 for P2 while stimulating S2 (amplitudes ranging from $-260 \mu \mathrm{V}$ to $-310 \mu \mathrm{V}, 20 \mathrm{~ms}$ delay) and S1 ( $-24 \mu \mathrm{V}, 38 \mathrm{~ms}$ delay). EP following S1 stimulation is a CCEP because of the presence of the Sylvian fissure and the operative cavity between the stimulation and recording sites. EPs following S2 are likely DCRs as they are recorded on the same gyrus, which is corroborated by their short latencies and maximized amplitudes with regard to the CCEP. Note the dashed lines indicating different amplitude scales for the DCRs, which were reduced by a factor 5 for visualization purposes.

\section{Discussion}

Variations in amplitude and delay of EPs are most likely due to different propagation mechanisms, which can be intra- or sub- cortical, and correspond to previously described DCRs and CCEPs. DCRs were not necessarily recorded in the immediate vicinity of the stimulation site (less than $2 \mathrm{~mm}$ as stated by Goldring et al. 1961) and can be observed at greater distances, as long as both the stimulation and recording are performed on the same gyrus. The electrode may thus detect the intracortically propagated response initiated at the stimulation site. By contrast and by definition, CCEP should be observed when DES and recording sites are not in the same gyrus. CCEP recorded in this experiment were delayed and attenuated in comparion with DCRs. The attenuation for CCEPs is probably the result of the limited temporal and spatial summation of post-synaptic potentials (PSPs) consequent to the divergence of white matter pathways arising out of the distant stimulation site. This contrasts with DCR, which involves the synchronous summation of PSPs in the vicinity of DES. Increased delays, on the other hand, may be caused by extra propagation time and be indicative of the conductive properties of the [short] association fibers being recruited. Unusually delayed EPs noticed for P2 when stimulating and recording the superior temporal gyrus may be evocative of pathologically altered fibers (but subcortical intragyral pathways are unknown) or implies intracortical propagation of DCRs which may be delayed and attenuated with distance. These late responses may also be interpreted as further integrative processes. Particular attention should be paid to the delays and amplitudes of EPs when the recording is performed over adjacent gyrus, because of the possible overlap of short latency CCEPs, propagated along intergyral association U-fibers, and DCR, elicited because of local electric conduction of the tissue. In such situation, it could be difficult to disentangle DCRs from short CCEPs. However, there are no such ambiguities with the EPs we presented as the stimulation and recording sites are on different lobes when measuring CCEPs. As a whole, differentiating CCEP from DCR based on anatomical landmarks is problematic. Due to a limited spatial resolution of most recording systems and methods, it is somehow difficult to identify the exact generator mechanisms of the evoked responses that may combine together. In consequence, the nomenclature for responses adjacent to the DES site is not yet totally clear. Indeed, they are also sometimes called "adjacent large CCEP responses" (Shimada et al. 2017, Kobayashi et al. 2017) while some prefer the classic term DCR.

All EPs we measured were very similar in shape, suggesting stereotyped electrophysiological response triggered by massive and synchronous activation rather than genuine output integration. The summation of PSPs is the key element shaping ECoG signal and appears loosely dependent of that occurring in input. According to previous works focusing on the peripheral system, the IED and PW were tuned in order for the electrical field to reach deeper and bigger neural elements in the cortical column and facilitate the generation of action potentials with less charge injections, namely to improve the generation of CCEP 
(Vincent et al. 2017). We observed a few differences depending on DES parameters, especially when using $1 \mathrm{~cm}$ IED, which produced more consistent and earlier, but altered in amplitude, CCEPs. This is in adequacy with some preliminary results showing that increased IED may elicit the P0 component of the DCR, suggesting a greater spatio-temporal summation of action potentials propagating on sub-cortical association fibers (Boyer et al. 2018).

The use of a 9-10 Hz stimulation may prevent the recording of later components which are observed in more recent CCEP studies performed on epileptic patients and using $1 \mathrm{~Hz}$ stimulation (Matsumoto et al. 2004, Yamao et al. 2014). However and importantly, there are major differences in DES parameters between these studies and older ones (which focused on DCR), and ours (Vincent et al. 2016, 2017). This is especially true when considering the current intensity. In our study we wanted to investigate whether classical N1 components could be observed with lower intensity of DES in order to use these electrophysiological responses in real-time, as putative indicators of connectivity and perform electrophysiological mapping during awake brain surgery. Interestingly, Goldring et al. (1961) demonstrated that the shape of DCR was not altered when DES frequency was inferior to $20 \mathrm{~Hz}$, but a slownegativity appeared (whose frequency was inferior to $1 \mathrm{~Hz}$ ). As for our data, we verified the stability of EPs over the stimulation period comparing subsets of averages.

From a methodological perspective, differential configuration allowed the recording of signals with better signal-to-noise ratio and more localized detection, at the expense of an unconventional shape of EPs. It remains particularly difficult to control for the electronic noise in such a neurosurgical context and a large part of the data has to be discarded. Nonetheless, it seems possible to discriminate DCRs from CCEPs and explore anatomofunctional connectivity using such configuration. We were, for instance, able to observe the bidirectional nature of the arcuate fasciculus as described in Matsumoto et al. (2004).

It is important to note that these measurements were performed on patients under general anesthesia, at the end of the surgery. Anesthesia may play some role on the excitability level and on the shape of the response. More specifically, it has been shown that drugs may be correlated with an increase of the N1 component and the disappearance of the (rare) N2 component (Goldring et al. 1961). However, the latencies and the amplitude of the N1 responses were not modified consistently by drugs injection.

\section{Conflict of interest statement}

On behalf of all authors, the corresponding author states that there is no conflict of interest.

\section{Sponsorships and funding}

Research supported by the LabEx NUMEV project ( ${ }^{\circ}$ ANR-10-LABX-20) funded by the French government's "Investissements d'Avenir" program managed by the French National Research Agency (ANR) and complementary grants from the Institut Universitaire de France and INSERM laboratory (U1093).

\section{References}


Adrian E (1936). "The spread of activity in the cerebral cortex" In: J Physiol, 88(2), pp. $127-161$.

Boyer A, H Duffau, M Vincent, S Ramdani, E Mandonnet, D Guiraud and F Bonnetblanc (2018). "Electrophysiological Activity Evoked by Direct Electrical Stimulation of the Human Brain: Interest of the P0 Component" In: Conf Proc IEEE Eng Med Biol Soc., pp. 2210-2213.

Duffau H (2015). "Stimulation mapping of white matter tracts to study brain functional connectivity" In: Nat Rev Neurol 11, pp. 255-265.

Goldring S, Jerva MJ, Holmes TG, O’Leary JL, Shields JR (1961). "Direct response of human cerebral cortex" In: Arch Neurol; 4:22-30.

Goldring S, Harding GW, Gregorie EM (1994). "Distinctive electrophysiological characteristics of functionally discrete brain areas: a tenable approach to functional localization" In: J Neurosurg ; 80:701-9.

Kobayashi K, Matsumoto R, Matsuhashi M, Usami K, Shimotake A, Kunieda T, Kikuchi T, Yoshida K, Mikuni N, Miyamoto S, Fukuyama H, Takahashi R, Ikeda A (2017). "High frequency activity overriding cortico-cortical evoked potentials reflects altered excitability in the human epileptic focus." In: Clin Neurophysiol., vol. 128, no 9, pp.16731681 .

Li CL, Chou SN (1962). "Cortcial Intracellular Synaptic Potentials and Direct Cortical Stimulation?" In: J. Cell. Comp. Physiol., vol. 60, pp 1-16.

Mandonnet, E, P A Winkler, H Duffau (2010). "Direct electrical stimulation as an input gate into brain functional networks: principles, advantages and limitations." In: Acta Neurochir (Wien), 152, 185-193.

Shimada S, Kunnii N, Kawai K, Matsuo T, Ishishita Y, Ibayashi K, Saito N (2017). "Impact of volume-conducted potential in interpretation of cortico-cortical evoked potential: Detailed analysis of high-resolution electrocorticography using two mathematical approaches.” In: Clin. Neurophysiol., vol. 128, no. 4, pp. 549-557.

Matsumoto R, DR Nair, E LaPresto, I Najm, W Bingaman, H Shibasaki, HO Lüders (2004). "Functional connectivity in the human language system: a cortico-cortical evoked potential study" In: Brain, vol. 127, no. 10, pp. 2316-2330.

Vincent M, D Guiraud, H Duffau, E Mandonnet, F Bonnetblanc (2017) "Electrophysiological brain mapping: Basics of recording evoked potentials induced by electrical stimulation and its physiological spreading in the human brain" In: Clin. Neurophysiol., vol. 128, no. 10, pp. 1886-1890.

Vincent M, O Rossel, M Hayashibe, G Herbet, H Duffau, D Guiraud, F Bonnetblanc (2016), "The difference between electrical microstimulation and direct electrical stimulation towards new opportunities for innovative functional brain mapping?" In: Rev. Neurosci., vol. 27, no. 3, pp. 231-258.

Yamao Y, Matsumoto R, Kunieda T, Arakawa Y, Kobayashi K, Usami K, Shibata S, Kikuchi T, Sawamoto N, Mikuni N, Ikeda A, Fukuyama H, Miyamoto S (2014). "Intraoperative dorsal language network mapping by using single-pulse electrical stimulation" In: Hum Brain Mapp;35 (9):4345-61. 